\title{
Avoidable paths in graphs
}

Marthe Bonamy*

CNRS, LaBRI

Université de Bordeaux

Bordeaux, France

marthe. bonamy@u-bordeaux.fr

\author{
Oscar Defrain ${ }^{\dagger}$ \\ LIMOS \\ Université Clermont Auvergne \\ Clermont-Ferrand, France \\ oscar.defrain@uca.fr \\ Jocelyn Thiebaut \\ LIFO \\ Université d'Orléans \\ Orléans, France \\ jocelyn.thiebaut@univ-orleans.fr
}

\author{
Meike Hatzel ${ }^{\ddagger}$ \\ Logik und Semantik \\ Technische Universität Berlin \\ Berlin, Germany \\ meike.hatzel@tu-berlin.de
}

Submitted: Sep 23, 2019; Accepted: Oct 18, 2020; Published: Dec 11, 2020

(C) The authors. Released under the CC BY-ND license (International 4.0).

\begin{abstract}
We prove a recent conjecture of Beisegel et al. that for every positive integer $k$, every graph containing an induced $P_{k}$ also contains an avoidable $P_{k}$. Avoidability generalises the notion of simpliciality best known in the context of chordal graphs. The conjecture was only established for $k \in\{1,2\}$ (Ohtsuki et al. 1976, and Beisegel et al. 2019, respectively). Our result also implies a result of Chvátal et al. 2002, which assumed cycle restrictions. We provide a constructive and elementary proof, relying on a single trick regarding the induction hypothesis. In the line of previous works, we discuss conditions for multiple avoidable paths to exist.
\end{abstract}

Mathematics Subject Classifications: 05C38, 05C60

\section{Introduction}

A graph $G$ is chordal if every induced cycle is of length three. A classical result of Dirac [Dir61] states that every chordal graph has a simplicial vertex, that is, a vertex

\footnotetext{
* Supported by the ANR project GrR ANR-18-CE40-0032.

†Supported by the ANR project GraphEn ANR-15-CE40-0009.

$\ddagger$ Supported by the European Research Council (ERC) under the European Union’s Horizon 2020 research and innovation programme (ERC Consolidator Grant DISTRUCT, grant agreement No 648527).
} 
whose neighbourhood is a clique. However, not all graphs exhibit the nice structure of chordal graphs, and the statement does not extend to general graphs.

\subsection{From simplicial vertices to avoidable paths}

One way to generalise Dirac's result is through the following more flexible notion.

Definition 1 (Avoidable vertex). A vertex $v$ in a graph $G$ is avoidable if every induced path on three vertices with middle vertex $v$ is contained in an induced cycle in $G$.

Note that in a chordal graph, every avoidable vertex is simplicial. The next theorem can be inferred from [OCF76, BB98, ACTV15]; see also $\left[\mathrm{BCG}^{+} 19\right]$ for a nice introduction.

Theorem 2. Every graph has an avoidable vertex.

Recently in $\left[\mathrm{BCG}^{+} 19\right]$, the authors considered a generalisation of the concept of avoidable vertices to edges, and extended Theorem 2 to that notion.

Definition 3 (Avoidable edge). An edge $u v$ in a graph $G$ is avoidable if every induced path on four vertices with middle edge $u v$ is contained in an induced cycle in $G$.

Theorem 4 (Beisegel et al. $\left.\left[\mathrm{BCG}^{+} 19\right]\right)$. Every graph that has an edge has an avoidable edge.

This notion naturally generalises to paths, as follows.

Definition 5 (Extension). Given an induced path $P$ in a graph $G$, an extension of $P$ is an induced path $x P y$ in $G$ for some vertices $x, y$.

Definition 6 (Failing). An induced path $P$ in a graph $G$ is failing if there is no induced cycle of $G$ containing $P$.

Definition 7 (Avoidable). A path $P$ in a graph $G$ is avoidable if it is induced and has no failing extension. Given a subgraph $G^{\prime}$ of $G$, we say that $P$ is an avoidable path of $G$ in $G^{\prime}$ if it is avoidable in $G$ and $V(P) \subseteq V\left(G^{\prime}\right)$.

A graph $G$ is $P_{k}$-free if it does not contain a $P_{k}$, that is, an induced path on $k$ vertices. In fact all paths considered in this paper are induced unless specifically stated otherwise. In $\left[\mathrm{BCG}^{+} 19\right]$ the authors conjecture that for every positive integer $k$, every graph either is $P_{k}$-free or contains an avoidable path on $k$ vertices. This conjecture is motivated by the following result of Chvátal et al. [CRS02], which generalises Dirac's theorem. A $C_{\geqslant p}$-free graph is a graph where every induced cycle has at most $p-1$ vertices. The $C_{\geqslant 4}$-free graphs are exactly the chordal graphs. Unless specified otherwise, we consider cycles to be induced.

Theorem 8 (Chvátal et al. [CRS02]). For every positive integer $k$, every $C_{\geqslant k+3}$-free graph either is $P_{k}$-free or contains an avoidable path on $k$ vertices. 
In fact, Theorem 8 originally states the existence of a simplicial path in the class of $C_{\geqslant k+3}$-free graphs. A simplicial path is an induced path with no extension: it is avoidable by vacuity. Note that these two definitions coincide in such a class, as no cycle on at most $k+2$ vertices can contain the extension of an induced path on $k$ vertices.

Here, we confirm the aforementioned conjecture $\left[\mathrm{BCG}^{+} 19\right.$, Conjecture 1], as follows.

Theorem 9. For every positive integer $k$, every graph either is $P_{k}$-free or contains an avoidable $P_{k}$.

In fact, we prove Theorem 9 using a stronger induction hypothesis, in the exact same flavour as [CRS02], see Theorem 17 in Section 2.

\subsection{Consequences}

We point out that the proof of Theorem 9 is self-sufficient, thus this supersedes the arguments for theorems 2, 4 and 8 .

By using ingredients of Theorem 17 (namely Lemma 15), we obtain a way to build more than one avoidable $P_{k}$. The following corollary follows from Lemma 16 and Theorem 17 .

Corollary 10. For every positive integer $k$, graph $G$ and subset $X \subseteq V(G)$ such that $G[X]$ is connected, either $G-N[X]$ is $P_{k}$-free or there is an avoidable $P_{k}$ of $G$ in $G-N[X]$.

Two paths $Q_{1}$ and $Q_{2}$ are adjacent if there is an edge between a vertex of $Q_{1}$ and a vertex of $Q_{2}$.

Corollary 11. For every positive integer $k$ and graph $G$, either $G$ does not contain two non-adjacent $P_{k}$, or it contains two non-adjacent avoidable $P_{k}$.

Proof. Let $Q_{1}$ and $Q_{2}$ be two non-adjacent $P_{k}$. By Corollary 10, either $G-N\left[Q_{1}\right]$ is $P_{k}$-free or there is an avoidable $P_{k}$ of $G$ in $G-N\left[Q_{1}\right]$. The first outcome is ruled out by the existence of $Q_{2}$. Let $Q_{2}^{\prime}$ be an avoidable $P_{k}$ of $G$ in $G-N\left[Q_{1}\right]$. We repeat the argument with $Q_{2}^{\prime}$ instead of $Q_{1}$, and obtain an avoidable $P_{k}$ of $G$ in $G-N\left[Q_{2}^{\prime}\right]$, call it $Q_{1}^{\prime}$. The two paths $Q_{1}^{\prime}$ and $Q_{2}^{\prime}$ are two non-adjacent avoidable $P_{k}$, as desired.

We can also wonder:

Question 12. For every positive integer $k$, does every graph $G$ either not contain two disjoint $P_{k}$, or contain two disjoint avoidable $P_{k}$ ?

We know the answer to be positive in the case $k \in\{1,2\}$, due to $\left[\mathrm{BCG}^{+} 19\right.$, Theorems 3.3 and 6.4]. The answer turns out to be negative in all other cases, as exhibited in the following counterexample for $k \geqslant 3$, which consists of a cycle on $2 k-1$ vertices with an added vertex adjacent to two consecutive vertices on the cycle (see Figure 1 for the case $k=3$ ). This graph contains two disjoint $P_{k}$, and it has $2 k$ vertices, so the vertex sets of any two disjoint $P_{k}$ are in fact complementary in the graph. Suppose that the graph contains two disjoint avoidable $P_{k}$, and note that each intersects the triangle (otherwise the complement would not be a path). Since there are three vertices in the triangle, 


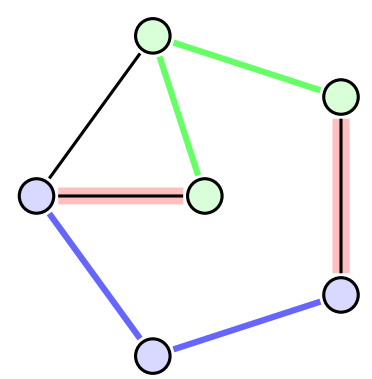

Figure 1: A graph that contains two disjoint $P_{3}$ (in blue and in green) but no two disjoint avoidable $P_{3}$ (there is a unique partition into two disjoint $P_{3}$, up to symmetry). In red, a failing extension of the blue path.

there is an avoidable $P_{k}$ containing a single vertex in the triangle. This $P_{k}$ has a failing extension, a contradiction.

In Section 3, we present a concise algorithm which follows the proof of Theorem 17. As discussed there, the algorithm has complexity $O\left(n^{k+2}\right)$ which, while naive, is the right order of magnitude under ETH.

\section{A stronger induction hypothesis}

All graphs considered in this paper are finite, simple and undirected. Given a graph $G$, we denote by $V(G)$ its set of vertices, and by $E(G) \subseteq\{\{x, y\} \mid x, y \in V(G), x \neq y\}$ its set of edges. Edges are denoted by $u v$ (or $v u$ ) instead of $\{u, v\}$. If $u v$ is an edge, then we say that $u$ and $v$ are adjacent. Given a vertex $u$, the neighbourhood $N(u)$ of $u$ is the set of vertices of $G$ that are adjacent to $u$. The closed neighbourhood $N[u]$ of $u$ is the set $N(u) \cup\{u\}$. If $X \subseteq V(G)$, then we define $N[X]:=\bigcup_{x \in X} N[x]$ and $N(X):=N[X] \backslash X$. The subgraph of $G$ induced by $X$, denoted by $G[X]$, is the graph $(X, E(G) \cap\{\{x, y\} \mid x, y \in X, x \neq y\})$, and $G-X$ is the graph $G[V(G) \backslash X]$. Given two adjacent vertices $u_{1}$ and $u_{2}$ of $G$, the graph obtained by merging $u_{1}$ and $u_{2}$ is the graph obtained from $G$ by replacing $u_{1}$ and $u_{2}$ with a new vertex $u$ such that $N(u)=N\left(\left\{u_{1}, u_{2}\right\}\right)$. Given a graph $G$ and two subsets $X$ and $Y$ of $V(G)$, we say that $X$ dominates $Y$ if every vertex of $Y \backslash X$ has a neighbour in $X$ (equivalently, if $Y \subseteq N[X]$ ).

We first define two useful properties.

Definition 13 (Basic property $\boldsymbol{H}_{\boldsymbol{B}}$ ). Given a positive integer $k$ and a graph $G$, the property $H_{B}(G, k)$ holds if either $G$ is $P_{k}$-free or there is an avoidable $P_{k}$ in $G$.

Definition 14 (Refined property $\boldsymbol{H}_{\boldsymbol{R}}$ ). Given a positive integer $k$, a graph $G$ and a vertex $u \in V(G)$, the property $H_{R}(G, k, u)$ holds if either $G-N[u]$ is $P_{k}$-free or there is an avoidable $P_{k}$ of $G$ in $G-N[u]$.

Given a positive integer $k$ and a graph $G$, the property $H_{R}(G, k)$ holds if $H_{R}(G, k, u)$ holds for every $u \in V(G)$. 
Note that property $H_{R}$ does not directly imply property $H_{B}$. We also emphasise the fact that an avoidable path of a subgraph is not necessarily an avoidable path of the whole graph.

We now prove a form of heredity in $H_{R}$.

Lemma 15. Let $k$ be a positive integer, $G$ a graph and $u_{1} u_{2}$ an edge of $G$. Let $G^{\prime}$ be the graph obtained from $G$ by merging the two vertices $u_{1}$ and $u_{2}$ into one vertex $u$. If $G^{\prime}-N[u]$ contains a $P_{k}$, then $H_{R}\left(G^{\prime}, k, u\right)$ implies $H_{R}\left(G, k, u_{1}\right)$.

Proof. Suppose $G^{\prime}-N[u]$ contains a $P_{k}$, and that $H_{R}\left(G^{\prime}, k, u\right)$ holds but not $H_{R}\left(G, k, u_{1}\right)$. Since $G^{\prime}-N[u]$ is not $P_{k}$-free, there is an avoidable $P_{k}$ of $G^{\prime}$ in $G^{\prime}-N[u]$. Call it $Q$. The path $Q$ is contained in $G^{\prime}-N[u]=G-N\left[\left\{u_{1}, u_{2}\right\}\right]$, so in particular in $G-N\left[u_{1}\right]$. Since $H_{R}\left(G, k, u_{1}\right)$ does not hold, $Q$ is not an avoidable $P_{k}$ of $G$. Thus, there is a failing extension $x Q y$ of $Q$ in $G$. Note that $x, y, u_{1}$, and $u_{2}$ are all pairwise distinct.

Hence, $x Q y$ is an extension of $Q$ in $G^{\prime}$, and, since $Q$ is avoidable in $G^{\prime}$, there is an induced cycle $C$ in $G^{\prime}$ containing the path $x Q y$. If $u \notin V(C)$, then the cycle $C$ is also an induced cycle in $G$ containing $x Q y$, a contradiction. Therefore, $u \in V(C)$. By replacing $u$ with either $u_{1}, u_{2}$ or the edge $u_{1} u_{2}$ as appropriate, we obtain an induced cycle in $G$ containing $x Q y$, a contradiction.

This even holds if we consider a connected subset of vertices in a graph instead of a single edge.

Lemma 16. Let $G$ be a graph and $X$ a subset of its vertices such that $G[X]$ is connected and $G-N[X]$ contains a $P_{k}$. Let $G^{\prime}$ be the graph obtained from $G$ by merging all vertices of $X$ into a single vertex $x$. Then $H_{R}\left(G^{\prime}, k, x\right)$ implies that there is an avoidable $P_{k}$ of $G$ in $G-N[X]$.

Proof. Suppose $H_{R}\left(G^{\prime}, k, x\right)$ holds but there is no avoidable $P_{k}$ of $G$ in $G-N[X]$. Since $G^{\prime}-N[x]$ is not $P_{k}$-free, there is an avoidable $P_{k}$ of $G^{\prime}$ in $G^{\prime}-N[x]$, call it $Q$, that is not an avoidable $P_{k}$ of $G$. Thus, there is a failing extension $a Q b$ of $Q$ in $G$. Note that $a, b$, and $x$ are all pairwise distinct.

Hence, $a Q b$ is an extension of $Q$ in $G^{\prime}$, and, since $Q$ is avoidable in $G^{\prime}$, there is an induced cycle $C$ in $G^{\prime}$ containing the path $a Q b$. If $x \notin V(C)$, then the cycle $C$ is also an induced cycle in $G$ containing $a Q b$, a contradiction. Therefore, $x \in V(C)$. Now let $a^{\prime}, b^{\prime}$ be the two neighbours of $x$ on $C$. By replacing $a^{\prime} x b^{\prime}$ in $C$ by a shortest $a^{\prime}-b^{\prime}$-path within $X$ (which exists because $G[X]$ is connected) we obtain an induced cycle $C^{\prime}$ in $G$ containing $a Q b$, a contradiction.

We are now ready to prove the main technical result of this paper.

Theorem 17. For every positive integer $k$ and every graph $G$, both properties $H_{B}(G, k)$ and $H_{R}(G, k)$ hold.

Proof. Suppose the statement is false and consider a counterexample $G$ which is minimal with respect to the number of vertices. 
Lemma 18. The property $H_{R}(G, k)$ holds for every $k$.

Proof. We proceed by contradiction. Suppose that $H_{R}(G, k, u)$ does not hold for some $k$ and some vertex $u \in V(G)$, that is, there exists a $P_{k}$ in $G-N[u]$, and every $P_{k}$ in $G-N[u]$ has a failing extension in $G$. We prove the following.

Claim 19. Every $P_{k}$ in $G-N[u]$ dominates $N(u)$.

Proof. Assume towards a contradiction that there is a $P_{k}$ in $G-N[u]$, call it $Q$, which is not adjacent to some vertex $v \in N(u)$. Then $G-N[\{u, v\}]$ contains a $P_{k}$. Let $G^{\prime}$ be the graph obtained from $G$ by merging $u$ and $v$ into a vertex $u^{\prime}$. Since $G^{\prime}$ has fewer vertices than $G$, property $H_{R}\left(G^{\prime}, k, u^{\prime}\right)$ holds by minimality of $G$. Then, by Lemma 15 , also $H_{R}(G, k, u)$ holds, a contradiction.

Let $G^{\prime}:=G-N[u]$. Then $G^{\prime}$ contains a $P_{k}$. As $G^{\prime}$ contains fewer vertices than $G$, the property $H_{B}\left(G^{\prime}, k\right)$ holds. Let $Q$ be an avoidable $P_{k}$ of $G^{\prime}$. By assumption, $Q$ is not an avoidable $P_{k}$ of $G$. So there is a failing extension $x Q y$ of $Q$ in $G$. Since $Q$ has no failing extension in $G^{\prime}$, we can assume without loss of generality that $y \in N(u)$. It follows that $x \notin N(u)$ : otherwise the cycle $x Q y u$ contradicts the fact that $x Q y$ is failing. By definition of an extension, $x Q y$ is an induced path. Let $z$ be the only neighbour of $y$ in $Q$, and let us now consider the path $x Q-z$ (which is the path obtained from $Q$ by first removing $z$ from one end and then adding $x$ to the other end). It is a $P_{k}$, and it does not intersect $N[u]$. However, no vertex in it is adjacent to $y$ which lies in $N(u)$, contradicting Claim 19.

Lemma 20. The property $H_{B}(G, k)$ holds for every $k$.

Proof. Assume towards a contradiction that for some $k$, property $H_{B}(G, k)$ does not hold. By Lemma 18, the property $H_{R}(G, k, u)$ holds for every vertex $u \in V(G)$. In other words, the graph $G$ contains a $P_{k}$ but no avoidable $P_{k}$, and for every vertex $u \in V(G)$, either $G-N[u]$ is $P_{k}$-free or there is an avoidable $P_{k}$ of $G$ in $G-N[u]$.

We derive the following claim.

Claim 21. Every $P_{k}$ in $G$ dominates $V(G)$.

Proof. Suppose there is a $P_{k}$, call it $Q$, that does not dominate some vertex $u$ of $G$. Since $H_{R}(G, k)$ holds, either $G-N[u]$ is $P_{k}$-free or there is an avoidable $P_{k}$ of $G$ in $G-N[u]$. The first case contradicts the existence of $Q$, and the second contradicts the fact that $H_{B}(G, k)$ does not hold.

Since $H_{B}(G, k)$ does not hold, $G$ contains a $P_{k}$, say $Q$, that is not avoidable. So it has a failing extension $x Q y$. Let $z$ be the only neighbour of $y$ in $Q$, and consider the path $x Q-z$. It is an induced $P_{k}$ and none of its vertices is adjacent to $y$. This contradicts Claim 21.

Finally, lemmas 18 and 20 together contradict $G$ being a counterexample.

Theorem 9 directly follows from Theorem 17. 


\section{An algorithm for Theorem 17}

By going through the proof and extracting the key ingredients, we obtain a straightforward algorithm verifying both properties (see Algorithm 1).

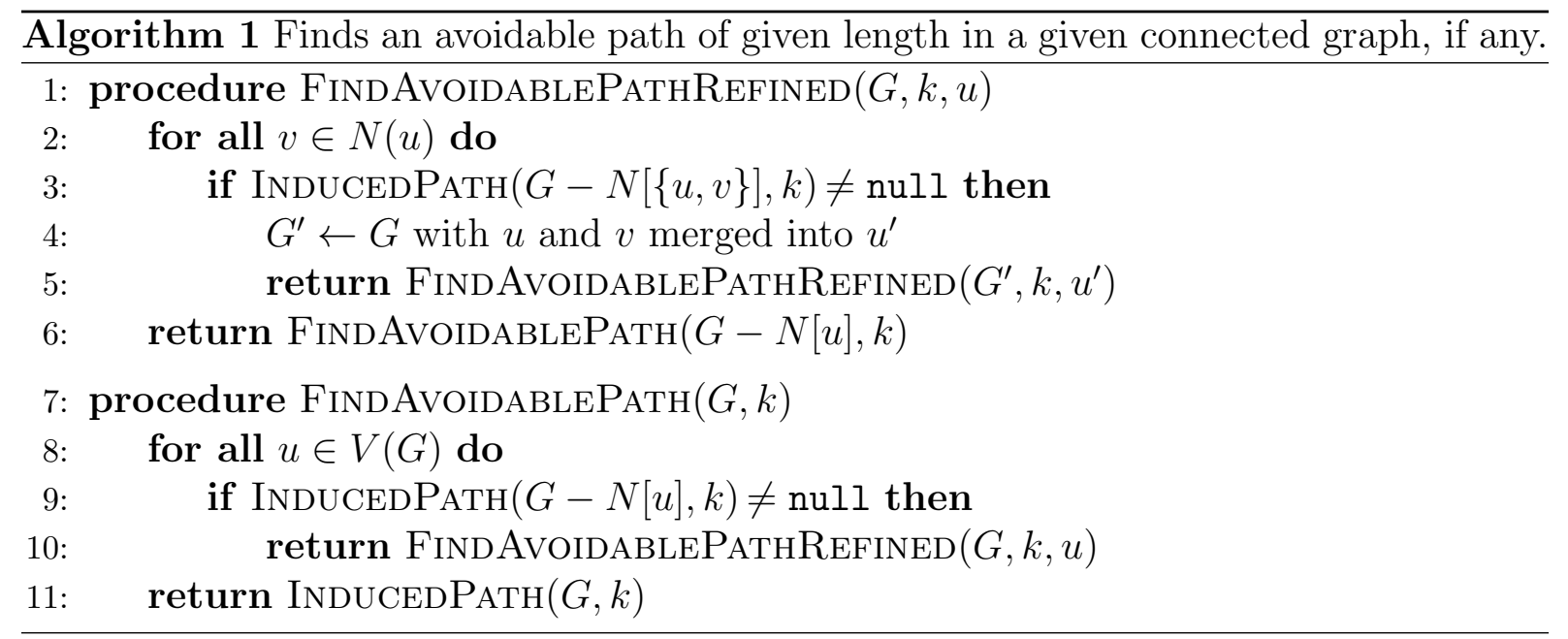

It suffices to consider connected graphs here, because if the graph is not connected, then its components can be computed in linear time and the algorithm can be run on the components separately. The algorithm uses the subprocedure INDUCEDPATH that, given a graph $G$ and a positive integer $k$, decides whether $G$ contains a $P_{k}$. If it does, the procedure returns a $P_{k}$, otherwise it returns null. The naive algorithm for that (testing all subsets of size $k$ ) has complexity $O\left(n^{k}\right)$. However, this is nearly optimal. Indeed, the problem of finding a $P_{k}$ in a given graph is $\mathrm{W}[1]$-hard ${ }^{1}$ when parametrised by $k$ (see $\left[\mathrm{CFK}^{+} 15\right.$, Ex. 13.16, p. 460]). In fact, the hinted reduction has a linear blow-up, so it follows that there is no $f(k) \cdot n^{o(k)}$ algorithm under ETH.

Let $k$ be a positive integer, then for $n \in \mathbb{N}^{+}$we define $B_{k}(n)\left(\operatorname{resp} . R_{k}(n)\right)$ to be the worst case complexity of FindAvoidablePath (resp. FindAvoidablePATHRefined) on an $n$-vertex graph with parameter $k$. We have $B_{k}(n) \leqslant n \cdot n^{k}+\max \left(R_{k}(n), n^{k}\right)$, and $R_{k}(n) \leqslant n \cdot n^{k}+\max \left(R_{k}(n-1), B_{k}(n-1)\right.$ ) (here the recursive instances are smaller by one when merging two vertices and smaller by at least 2 , when removing the closed neighbourhood because in a connected graph every vertex has at least one neighbour). We obtain $R_{k}(n) \in \mathcal{O}\left(n^{k+2}\right)$ and $B_{k}(n) \in \mathcal{O}\left(n^{k+2}+n^{k+1}\right)$. While this may well be improved, the known limitations for finding an induced path on $k$ vertices also apply for an induced avoidable path on $k$ vertices (by Theorem 9, if the first exists, then so does the second). Therefore, the order of magnitude of this naive algorithm is correct.

Note that there is a yet more naive algorithm blindly checking for every subset of size $k$ if it corresponds to an avoidable path. That algorithm has comparable complexity to ours (though slightly worse, at least at first sight). However, we wanted to emphasise that our proof of Theorem 17 is constructive and yields an elementary algorithm. Also, we believe that it provides an outline of the proof, which might be helpful to the reader.

\footnotetext{
${ }^{1}$ see e.g. $\left[\mathrm{CFK}^{+} 15\right]$ for definitions around complexity
} 


\section{Conclusion}

Given the discussions in Section 1.2, it is tempting to ask when a graph admits three (or more) disjoint (resp. pairwise non-adjacent) avoidable paths. Note that though Corollary 10 arms us with sufficient conditions for there to be more than two avoidable $P_{k}$, we do not believe that the corresponding sufficient conditions are necessary. However, it seems the picture is murky already for chordal graphs.

It is tempting to wonder whether we can obtain another avoidable structure. Though in some cases the very notion of extension becomes unclear (what should an extension of a clique be?), it does not seem like any other structure survives the test of chordal graphs or simple ad hoc constructions - even when allowing a family of graphs instead of fixing a single pattern (like a path on $k$ vertices). This motivates us to formulate the following question.

Question 22. Does there exist a family $\mathcal{H}$ of connected graphs, not containing any path, such that any graph is either $\mathcal{H}$-free or contains an avoidable element of $\mathcal{H}$ ?

The notion of avoidability in this context is deliberately left up to interpretation.

\section{Acknowledgements}

We gratefully acknowledge support from Nicolas Bonichon and the Simon family for the organisation of the $4^{\text {th }}$ Pessac Graph Workshop, where this research was done. We are indebted to Michał Pilipczuk for providing helpful references regarding the complexity of finding an induced path of given length. Last but not least, we thank Peppie for her unwavering support during the work sessions.

\section{References}

[ACTV15] Pierre Aboulker, Pierre Charbit, Nicolas Trotignon, and Kristina Vušković. Vertex elimination orderings for hereditary graph classes. Discrete Mathematics, 338(5):825-834, 2015.

[BB98] Anne Berry and Jean-Paul Bordat. Separability generalizes Dirac's theorem. Discrete Applied Mathematics, 84(1-3):43-53, 1998.

$\left[\mathrm{BCG}^{+} 19\right]$ Jesse Beisegel, Maria Chudnovsky, Vladimir Gurvich, Martin Milanič, and Mary Servatius. Avoidable vertices and edges in graphs. In Zachary Friggstad, Jörg-Rüdiger Sack, and Mohammad R Salavatipour, editors, Algorithms and Data Structures, pages 126-139, Cham, 2019. Springer International Publishing.

[CFK $\left.{ }^{+} 15\right]$ Marek Cygan, Fedor V Fomin, Łukasz Kowalik, Daniel Lokshtanov, Dániel Marx, Marcin Pilipczuk, Michał Pilipczuk, and Saket Saurabh. Parameterized algorithms. Springer, 2015. 
[CRS02] Vašek Chvátal, Irena Rusu, and R. Sritharan. Dirac-type characterizations of graphs without long chordless cycles. Discrete Mathematics, 256(1-2):445-448, 2002.

[Dir61] Gabriel Andrew Dirac. On rigid circuit graphs. Abhandlungen aus dem Mathematischen Seminar der Universität Hamburg, 25(1):71-76, 1961.

[OCF76] Tatsuo Ohtsuki, Lap Kit Cheung, and Toshio Fujisawa. Minimal triangulation of a graph and optimal pivoting order in a sparse matrix. Journal of Mathematical Analysis and Applications, 54(3):622-633, 1976. 